\title{
Glycerol-Silicone Membranes for Sustained and Controlled Topical Delivery of Antimicrobial and PainRelief Drugs
}

Mazurek, Piotr; Frederiksen, Nikoline S.; Silau, Harald; Yuusuf, Nuura A.; Mordhorst, Hanne; Pamp, Sünje J.; Skov, Anne L.

Published in:

Advanced Materials Interfaces

Link to article, DOI:

10.1002/admi.202001873

Publication date:

2021

Document Version

Peer reviewed version

Link back to DTU Orbit

Citation (APA):

Mazurek, P., Frederiksen, N. S., Silau, H., Yuusuf, N. A., Mordhorst, H., Pamp, S. J., \& Skov, A. L. (2021). Glycerol-Silicone Membranes for Sustained and Controlled Topical Delivery of Antimicrobial and PainRelief Drugs. Advanced Materials Interfaces, 8(5), [2001873]. https://doi.org/10.1002/admi.202001873

\section{General rights}

Copyright and moral rights for the publications made accessible in the public portal are retained by the authors and/or other copyright owners and it is a condition of accessing publications that users recognise and abide by the legal requirements associated with these rights.

- Users may download and print one copy of any publication from the public portal for the purpose of private study or research.

- You may not further distribute the material or use it for any profit-making activity or commercial gain

- You may freely distribute the URL identifying the publication in the public portal 


\title{
WILEY-VCH
}

\section{Glycerol-Silicone Membranes for Sustained and Controlled Topical Delivery of Antimicrobial and Pain-Relief Drugs}

\author{
Piotr Mazurek, Nikoline S. Frederiksen, Harald Silau, Nuura A. Yuusuf, Hanne Mordhorst, \\ Sünje J. Pamp, Anne L. Skov*
}

Piotr Mazurek, Nikoline S. Frederiksen, Harald Silau, Prof. Anne L. Skov

Danish Polymer Centre, Department of Chemical Engineering, Technical University of Denmark, 2800 Kgs. Lyngby, Denmark

E-mail: al@kt.dtu.dk

Prof. Anne L. Skov

Glysious Polymer Technologies, 2800 Kgs. Lyngby, Denmark

Nuura A. Yuusuf, Hanne Mordhorst, Assoc. Prof. Sünje J. Pamp,

Research Group for Genomic Epidemiology, National Food Institute, Technical University of Denmark, 2800 Kgs. Lyngby, Denmark

Keywords: wound care, dressing, silicone, glycerol, drug delivery

\begin{abstract}
Controlling drug delivery from medical dressings to wounds remains a challenge despite the presence of multiple drug-containing wound dressings on the market. The dressing should ideally be effective immediately after application and still show no burst effect, and must also be comfortable for an extended period of time. Here, a silicone-based membrane is shown to offer an adjustable, constant release of various drugs even at a minimum moisture level resembling that of dry wounds. The drugs are dissolved in glycerol, which is speed-mixed with silicone and subsequently cured to yield a solid membrane with evenly distributed glycerol microcontainers housing the drug. The drugs are released immediately upon contact with moisture, enabling efficient treatment with short response time and no burst. Drug delivery capability is evaluated using a classical drug release experiment, as well as by performing an antimicrobial susceptibility test on ten bacterial strains in agar diffusion assays. Results show that glycerol-silicone membranes are promising candidates for fighting bacterial infections. Moreover, the addition of glycerol domains to a silicone matrix is proven to have a positive impact on water vapor transmission rate and moisture handling capabilities, resulting in a more skin compliant dressing.
\end{abstract}




\section{WILEY-VCH}

\section{Introduction}

With a growing elderly population as well as increased obesity and related lifestyle diseases, wounds - and particularly chronic wounds - have become a serious socioeconomic burden. The importance of developing novel, improved dressings for treating complex wounds is thus undisputable, both from the patient's point of view and from that of the healthcare system. Complex wounds, often defined as acute and chronic wounds that are difficult to heal, affect the lives of millions of people. ${ }^{[1,2]}$ Indeed, in 2017 chronic wounds alone were reported to cause the highest burden to the healthcare system of all skin diseases in the United States, and account for as much as $3 \%$ of the healthcare budget in developed countries, making them a multibilliondollar global problem. ${ }^{[3-7]}$

Wound dressings' primary function is to protect the wound site from the outside environment and absorb wound exudate. However, because complex wounds usually require special treatment, dedicated dressings with more advanced functionality are needed. ${ }^{[8,9]}$ In addition to its basic functions, an advanced wound dressing should maintain a moist wound environment and ensure efficient gaseous exchange. ${ }^{[1,10,11]}$ The newest generation of dressings for complex wounds incorporate bioactive substances which are delivered to the wound site, and which include antimicrobial agents and anti-inflammatory drugs. ${ }^{[12-15]}$ Antimicrobial drugs delivered to the wound site have been proven to efficiently eliminate bacteria that would otherwise hamper proper wound healing. The major advantage of a topical over systemic administration of antimicrobials is a lower required drug dose, which is deemed crucial due to ameliorating increased bacterial resistance to antibiotics. ${ }^{[16-18]}$ In parallel, it is critical to ensure that the applied antibiotic is both suitable for the specific wound and delivered in a controlled way, so as to efficiently eliminate bacteria. ${ }^{[17,19,20]}$ Systemically and cutaneously administered antimicrobial drugs thus reduce the bacterial load while also lessening inflammation in the longer term. ${ }^{[8]}$ Because pain relief is not typically experienced until several days after administration of the antimicrobial drug, in the case of painful wounds it is recommended that 


\section{WILEY-VCH}

administration of pain-relief agents be prioritized equally with treatment of the infection. ${ }^{[8,21]}$ Ibuprofen is a commonly used anti-inflammatory drug which has been proven to effectively minimize the pain and inflammation associated with complex wounds. ${ }^{[22-25]}$ Locally applied anesthetic drugs—e.g., lidocaine — are also commonly used to minimize chronic wound pain. ${ }^{[8]}$ Silicones and silicone-based devices are gaining importance in the biomedical field ${ }^{[26]}$ due to their combination of excellent mechanical properties, breathability, chemical stability, and biocompatibility making silicone elastomers and adhesives important components of approved medical devices. ${ }^{[27-30]}$ Recently, numerous studies have investigated the use of silicones as adhesives and membranes for cutaneous wound treatment: e.g., state-of-the-art chronic wound dressings and fast clotting dressings. ${ }^{[30-32]}$ Extensive research has been conducted on enhancing the properties of silicones to produce improved components for novel wound dressings. ${ }^{[33-35]}$ Glycerol-silicone elastomers (GSEs) are two-phase materials originating from the combination of two virtually immiscible liquids into stable emulsions. GSEs are prepared in a one-step process in which a hydrophobic silicone prepolymer is mixed with a hydrophilic glycerol. Via the application of high shear forces, the glycerol phase is broken down to micrometer size droplets, forming a glycerol-in-silicone prepolymer emulsion. ${ }^{[36]}$ The silicone phase then crosslinks upon exposure to elevated temperatures, forming a free-standing hybrid elastomer. The preparation procedure is schematically presented in Figure 1. GSEs have recently been reported to allow for the controlled delivery of active substances ${ }^{[37]}$ dissolved in the glycerol droplets, which are released upon contact with an aqueous environment. Interestingly, this release can be tuned to follow first-, near zero- or zero-order kinetics. Near zero- and zero-order release kinetics are particularly valuable in wound care, as they allow a constant drug dose to be delivered to the wound. Importantly, GSEs can be shaped to form thin elastomeric membranes (down to around 20-30 $\mu \mathrm{m}$ ), bulk samples, foams and adhesives. ${ }^{[36,38-40]}$ Such versatility increases the spectrum of potential GSE applications. 


\section{WILEY-VCH}

GSEs have shown promise as a platform technology for delivering simple organic molecules. ${ }^{[37]}$ Here, we verified that more complex active substances such as common antimicrobial and antiinflammatory drugs can also be released effectively from the membranes. Ultimately, release efficiency was assessed by testing the membranes' antimicrobial activity. In order to fully explore the glycerol-silicone membranes' potential as candidates for components in wound dressings, their morphology, mechanical properties, water vapor transmission rate (WVTR) and fluid handling capabilities were also investigated.

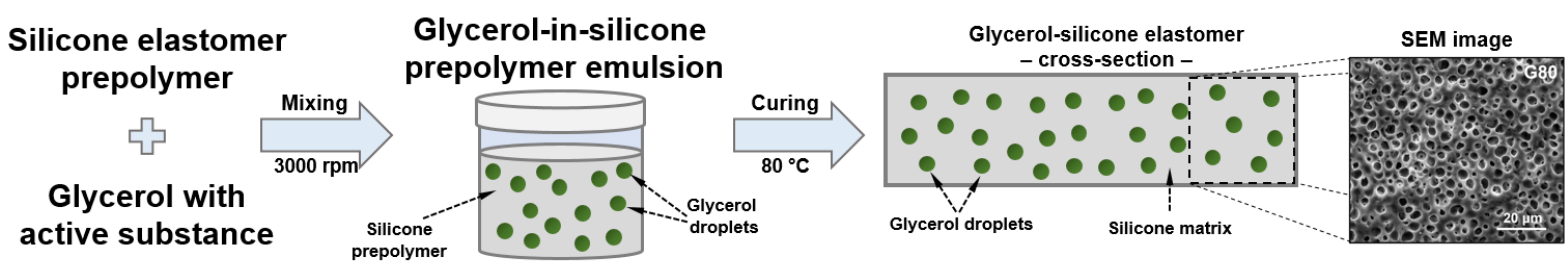

Figure 1. Schematic depiction of the preparation protocol for glycerol-silicone membranes containing active substances. Glycerol with pre-dissolved drug is speed-mixed with a silicone prepolymer; a stable emulsion is obtained by applying high shear forces to the mixture, which forms a dual-phase elastomer upon curing of the silicone phase.

\section{Experimental}

\subsection{Materials}

A two-component hydrosilylation-cured Sylgard 184 (S184) silicone kit was obtained from Dow, US. Glycerol (>99.5\% purity), tetracycline (TET), triclosan (TCS), lidocaine (LDC), ibuprofen (IBU), mupirocin (MUP), diclofenac sodium salt (DCF), benzalkonium chloride (BKC), $\beta$-cyclodextrin $(\beta C D)$, sodium chloride and human blood serum (from human male AB plasma, sterile-filtered) were purchased from Sigma Aldrich, Denmark. Karstedt's catalyst platinum(0) 1,3-divinyl-1,1,3,3-tetramethyldisiloxane complex, 2\% - was obtained from Gelest, 


\section{WILEY-VCH}

US. Stabilized artificial eccrine perspiration (artificial sweat) was obtained from LC Tech, Germany. Arclear 8932EE silicone transfer tape was kindly provided by Adhesives Research Inc., UK. Poly(ethylene terephthalate) (PET) Hostaphan RN film with a thickness of $190 \mu \mathrm{m}$ was purchased from Pütz-Folien, Germany. All chemicals and products were used as received. Commercial wound dressings, Biatain Alginate Ag (Coloplast A/S, Denmark) and Mepilex Ag (Mölnlycke Health Care, Sweden), were purchased in a local pharmacy. Agar was purchased from Oxoid, Spain. Lysogeny broth (LB) and brain heart infusion (BHI) broth were obtained from Sigma Aldrich, USA and Oxoid, UK, respectively.

\subsection{Sample preparation}

A specific amount of active substance was dissolved in glycerol at $60^{\circ} \mathrm{C}$. Because triclosan, mupirocin, ibuprofen and lidocaine are not soluble in glycerol, $\beta$-cyclodextrin $(\beta C D)$ was added to glycerol (different drug: $\beta C D$ molar ratios were used for different drugs, as listed in Table 1) to facilitate dissolution. After cooling, the homogenous solution was added to S184 (mixed in a ratio of 10:1, base:curing agent) and mixed for 2 minutes at 3500 revolutions per minute (rpm) using a dual asymmetric centrifuge SpeedMixer DAC 150 FVZ-K. The prepared stable, uniform emulsion was cast onto a PET film and coated using a commercial coating knife Elcometer 3540 Bird Film Applicator. The sample was subsequently cured for 1 hour at $80^{\circ} \mathrm{C}$. The specimens were stored at room temperature in airtight containers. As tetracycline, benzalkonium choride, diclofenac and lidocaine contain amines which are well-known inhibitors of the Pt-catalyzed curing reaction ${ }^{[27]}$, an additional $50 \mathrm{ppm}$ of the Pt catalyst was added to formulations containing these four drugs to enable proper cross-linking.

The term 'phr' denotes the weight parts of incorporated glycerol per hundred weight parts of the applied silicone rubber. The sample names were formed using the pattern GX, where G stands for glycerol and X corresponds to the glycerol in phr added to silicone. The sample code 
was additionally extended with drug acronyms followed by their respective concentrations in glycerol (see Table 1).

Table 1. List of investigated samples in chronological order of their occurrence in the manuscript.

\begin{tabular}{|c|c|c|c|c|c|c|}
\hline No & Sample name & $\begin{array}{c}\text { Glycerol } \\
\text { cont. [phr] }\end{array}$ & Incorporated drug & $\begin{array}{l}\text { Drug conc. in } \\
\text { glycerol [\%] }\end{array}$ & $\begin{array}{l}\text { Added } \beta C D \text { [molar } \\
\text { ratio drug: } \beta C D]\end{array}$ & $\begin{array}{l}\text { Added Pt } \\
\text { cat. [ppm] }\end{array}$ \\
\hline 1 & G0 & 0 & - & - & - & - \\
\hline 2 & G40 & 40 & - & - & - & - \\
\hline 3 & G80 & 80 & - & - & - & - \\
\hline 4 & G120 & 120 & - & - & - & - \\
\hline 5 & G80(TET-1) & 80 & Tetracycline & 1 & - & 50 \\
\hline 6 & G80(TET-0.5) & 80 & Tetracycline & 0.5 & - & 50 \\
\hline 7 & G80(TET-2) & 80 & Tetracycline & 2 & - & 50 \\
\hline 8 & G40(TET-1) & 40 & Tetracycline & 1 & - & 50 \\
\hline 9 & G100(TET-1) & 100 & Tetracycline & 1 & - & 50 \\
\hline 10 & G80(TCS-1) & 80 & Triclosan & 1 & $5: 1$ & - \\
\hline 11 & G80(MUP-1) & 80 & Mupirocin & 1 & $5: 1$ & - \\
\hline 12 & G80(BKC-1) & 80 & Benzalkonium chloride & 1 & - & 50 \\
\hline 13 & G80(IBU-0.5) & 80 & Ibuprofen & 0.5 & $1: 1$ & - \\
\hline 14 & G80(DCF-1) & 80 & Diclofenac sodium salt & 1 & - & 50 \\
\hline 15 & G80(LDC-0.5) & 80 & Lidocaine & 0.5 & $1: 1$ & 50 \\
\hline
\end{tabular}

\subsection{Methods}

Morphology of the glycerol-silicone elastomers was investigated using a FEI Quanta 200 ESEM FEG scanning electron microscope (SEM). Cressington 208 HR high-resolution sputter coater was applied to coat samples with a $2 \mathrm{~nm}$ thick layer of gold. Cross-sections of samples with and without incorporated drugs were investigated and compared.

Mechanical properties of membranes were investigated using an Instron 3345 tensile tester equipped with a $500 \mathrm{~N}$ load cell. Dog-bone samples (ASTM D412 type C) with a thickness of around 300 um were stretched with a crosshead velocity of $500 \mathrm{~mm} / \mathrm{min}$. Five specimens were tested for each formulation and results were averaged. 


\section{WILEY-VCH}

Water vapor transmission rate (WVTR) experiments were conducted using a protocol reported by Trifol et al. ${ }^{[41,42]}$ Membranes $\left(0.1 \mathrm{~mm}\right.$ thick) were conditioned at $23^{\circ} \mathrm{C}$ and $50 \%$ relative humidity (RH) for $12 \mathrm{~h}$ before measurement. No substantial water uptake by the membranes was observed during this period. A metal container was filled with silica gel (desiccant), which was pre-dried for $12 \mathrm{~h}$ at $120^{\circ} \mathrm{C}$. The membrane was tightly sealed over the open container, which was placed in a climate-controlled chamber set at $23^{\circ} \mathrm{C}$ and $50 \% \mathrm{RH}$. The mass increase of each individual setup (three specimens per composition) was monitored over 8 hours and the results were averaged.

Absorption of deionized (DI) water, artificial sweat and human blood serum was studied using a DataPhysics OCA20 goniometer. $30 \mu \mathrm{L}$ droplets were dispensed on silicone and glycerolsilicone membranes with a thickness of $300-400 \mu \mathrm{m}$, and the progress of fluid absorption was monitored for at least $7 \mathrm{~h}$. Each droplet placed on a sample was tightly sealed using a $20 \times 20$ x $20 \mathrm{~mm}$ cubical glass cell to minimize evaporation.

\section{Active substance release}

Samples with a thickness of around $60 \mu \mathrm{m}$ were transferred onto Arclear tape in order to permanently attach them to a stiffer support. Specimens with dimensions of $0.9 \times 5 \mathrm{~cm}$ were cut out and inserted into a quartz cuvette equipped with a magnetic stir bar and filled with $3 \mathrm{~mL}$ of DI water or $0.9 \mathrm{wt} \%$ sodium chloride solution (saline water). The cuvette was subsequently sealed with a septum and transferred into the spectrophotometer. Substance release progress was investigated by monitoring changes in absorbance over time using a Vernier UV-vis spectrophotometer. Each formulation was tested three times and results were averaged.

\section{Antimicrobial susceptibility assay}

Antimicrobial susceptibility of bacteria towards GSE membranes and two commercial wound dressings was tested in agar diffusion assays. The examination included eight bacterial species, represented by a total of ten strains, that are commonly found on the human skin and/or in acute and chronic wounds (see list below). All bacterial strains were cultivated on LB agar, except 


\section{WILEY-VCH}

Streptococcus pyogenes, which was cultivated on BHI agar. Standardized bacteria suspensions were prepared in $0.9 \% \mathrm{NaCl}$ solution with McFarland 0.5 turbidity and evenly distributed on agar plates (14 cm diameter) with cotton swabs. A maximum of five samples (GSE membranes or commercial wound dressings, $6 \mathrm{~mm}$ diameter) were placed onto the agar and evenly distributed to allow for the measurement of inhibition zones. The two commercially available wound dressings tested were Biatain Alginate Ag (a highly absorbent dressing for moderate-toheavily exuding infected wounds, containing silver sodium hydrogen zirconium phosphate complex as an antimicrobial substance) and Mepilex Ag (foam dressing for low-to-moderately exuding burns and wounds, containing silver sulfate as an antimicrobial substance). The plates were incubated at $35^{\circ} \mathrm{C}$ for $18-20$ hours, and the diameter of the growth-inhibition zone was measured. Triplicate agar plates were prepared for each bacterial strain, and each assay was performed three times (i.e., a total of nine measurements per bacterial strain and GSE membrane or commercial wound dressing). The ten strains tested were: Pseudomonas aeruginosa PAO1, Acinetobacter baumanii SJ531, Escherichia coli mcr-1 (NCTC 13846), Staphylococcus aureus NCTC 8325-4, Staphylococcus aureus NCTC 8325, Escherichia coli ATCC 29212, Enterococcus faecalis ATCC 29212, Staphylococcus epidermidis ARBAO 1-7 (STC-1.58), Streptococcus pyogenes ATCC 12385 and Klebsiella pneumoniae ATCC 700721. In addition, liquid-based assays were performed to examine bacterial susceptibility to the commercial wound dressings. Bacterial cultures were prepared with sterile tubes containing $4 \mathrm{~mL}$ LB medium (Sigma-Aldrich, USA) inoculated with A. baumanii to an optical density $\mathrm{OD}_{600}$ of 0.05 . A Mepilex sample (6 mm diameter) was then added to the culture. Cultures without Mepilex samples served as controls. Duplicate cultures were prepared for each setup and incubated at $35^{\circ} \mathrm{C}$ for $18-20$ hours. The $\mathrm{OD}_{600}$ was recorded for each culture tube.

\section{Results and discussion}




\section{WILEY-VCH}

\subsection{Mechanical properties of the glycerol-silicone membranes}

Glycerol-silicone membranes with a thickness of $300 \mu \mathrm{m}$ were exposed to mechanical deformation in order to evaluate their mechanical performance. The data presented in Figure 2 shows that the Young's modulus decreased from 1.17 MPa for the pure S184 to $0.68,0.56$ and $0.47 \mathrm{MPa}$ for compositions G40, G80 and G120, respectively, in line with the volumetric dilution of the silicone matrix. Because the glycerol droplets act as domains of zero-stress, the glycerol-silicone material is softer compared to the reference sample. Furthermore, the data indicates that both G40 and G80 samples exhibit similar strains at break of around 190\%, implying that maximum extensibility is not compromised when 40 and $80 \mathrm{phr}$ of glycerol is embedded into silicone elastomers. On the other hand, the sample with $120 \mathrm{phr}$ of glycerol exhibited a substantially lower strain at break. At high glycerol loadings, the silicone phase separating glycerol domains is too thin to provide non-compromised mechanical integrity under the large stresses, resulting in slightly weakened performance. However, this formulation's performance is still satisfactory considering the stress withstood by the silicone itself (see details in SI, section S2).

The theoretical values of the Young's modulus for each GSE was calculated, taking into consideration that the modulus of glycerol is negligible and assuming no significant contribution from interfacial energies (this assumption holds for silicone elastomers, but not, e.g., for silicone adhesives ${ }^{[40,43,44]}$ ). Furthermore, the modulus of the silicone phase is assumed to be equal to the modulus obtained experimentally for the pristine S184 formulation (see details in SI, section S2). Interestingly, the theoretical values $(0.88,0.71$ and $0.59 \mathrm{MPa}$ for samples G40, G80 and G120, respectively) are higher compared to the experimental results. This difference must be caused by either an extensive mobility at the glycerol-silicone interface, which minimizes the stress generated in the silicone matrix when mechanical deformation is imposed, or by a lower crosslinking degree at the surface due to two-dimensional crosslinking only. The Young's modulus and ultimate strain values of $300 \mu \mathrm{m}$ thick samples evaluated in 


\section{WILEY-VCH}

this study are comparable with the previously reported mechanical behavior of $3 \mathrm{~mm}$ thick specimens $^{[36]}$, confirming that glycerol-silicone elastomers possess excellent mechanical integrity over a broad range of sample thicknesses.

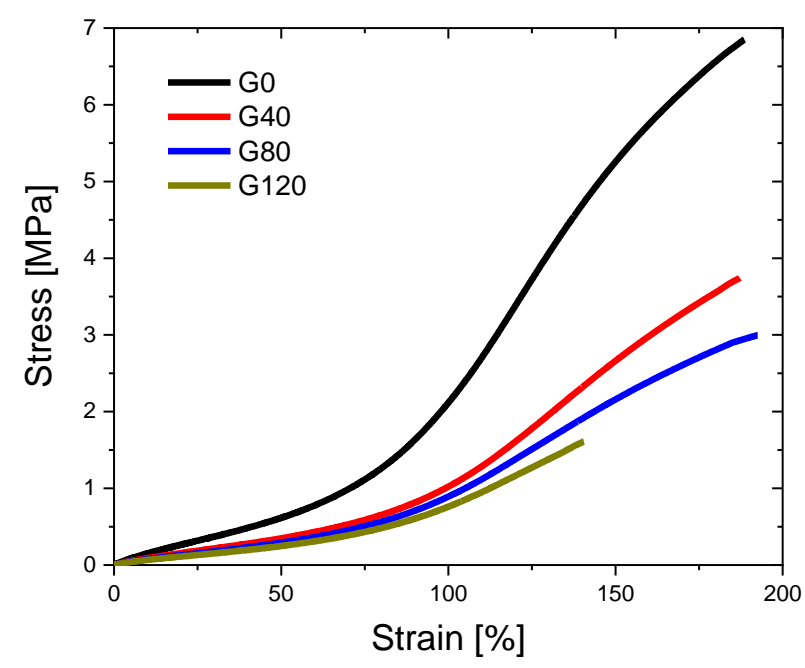

Figure 2. Stress-strain curves of a pure S184 sample and various glycerol-silicone formulations. Thickness of all the samples was around $300 \mu \mathrm{m}$. Tests were performed at room temperature and the applied crosshead velocity was $500 \mathrm{~mm} / \mathrm{min}$.

\subsection{Water vapor transmission rate (WVTR)}

Water vapor transmission is a key feature of wound dressings, so it is necessary to ensure that none of the dressing's components compromises this properityproperty. ${ }^{[8,30]}$ The WVTR was evaluated using the experimental setup presented graphically in Figure 3A , and was calculated from:

$$
W V T R=\frac{n l}{S}
$$




\section{WILEY-VCH}

where $n$ is the slope of the mass increase over time, while $l$ and $\mathrm{S}$ are the thickness and surface

area of the membrane, respectively. The recorded mass changes correspond to water absorption by the desiccant. Importantly, the mass increase of the tested membranes was marginal and did not influence the measurement. The averaged WVTR values of samples with various amounts of glycerol are presented in Figure 3B. The addition of 40 and $80 \mathrm{phr}$ of glycerol enhanced the WVTR compared to pure S184 by $67 \%$ and 96\%, respectively, while sample G120_S184 exhibited a $200 \%$ increase. Because water diffuses substantially faster through glycerol compared to silicone, the glycerol domains form zones of very high WVTR in the glycerolsilicone elastomers. Thus, higher glycerol loadings generally result in materials with more efficient water vapor transport.
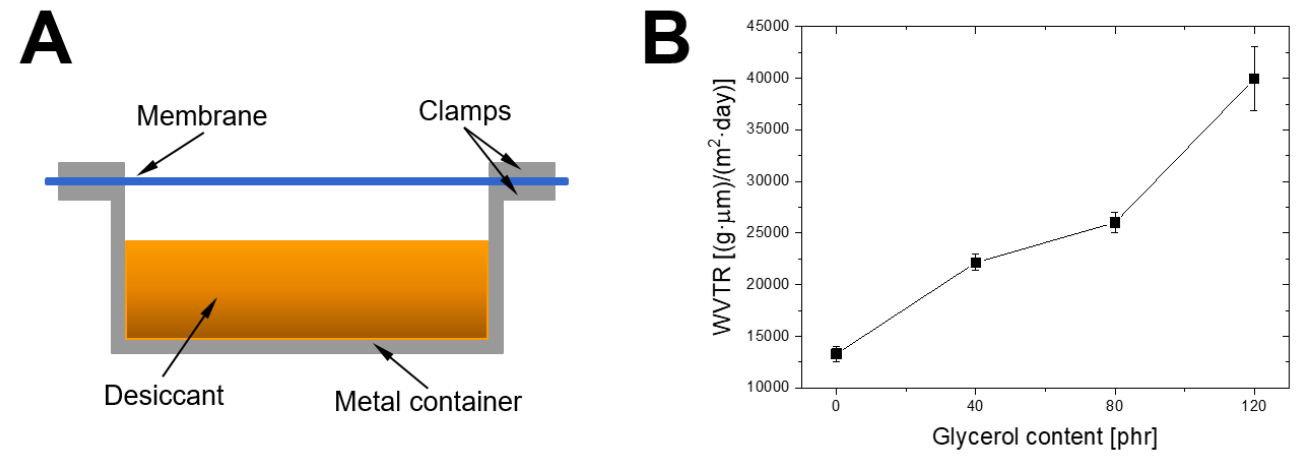

Figure 3. (A) Schematics of the experimental setup used to determine WVTR. Dried desiccant was placed in a metal cup, on top of which an investigated material was placed. (B) Determined WVTR of GSEs with various amounts of glycerol $(0,40,80$ and $120 \mathrm{phr})$ measured at $23^{\circ} \mathrm{C}$ and $50 \%$ relative humidity.

\subsection{Fluid absorption}

The fluid absorption capacity of various glycerol-silicone elastomers was investigated. Most silicone-based materials are strongly hydrophobic, and hence do not absorb hydrophilic liquids such as water. However, it was reported previously that glycerol-silicone elastomers can absorb 


\section{WILEY-VCH}

substantial amounts of water due to the hygroscopic nature of the incorporated glycerol. ${ }^{[36,37]}$ In this study, $30 \mu \mathrm{L}$ droplets of DI water, artificial sweat, and human blood serum were dispensed onto various membranes, and fluid absorption was monitored over 6 hours (the full data set is available in the Supporting Information, Figure S2). The obtained results clearly indicate that the glycerol-silicone membranes exhibit substantially increased absorption of hydrophilic liquids compared to the pure silicone, as shown in Figure 4. In other words, absorption capabilities improve with increased glycerol loading. For example, sample G120 absorbed nearly $100 \%$ of the $30 \mu \mathrm{L}$ of dispensed liquids in less than $3 \mathrm{~h}$. This proves that the GSE membranes allow for enhanced, controlled management of wound fluids and sweat released by the skin in the wound surroundings.
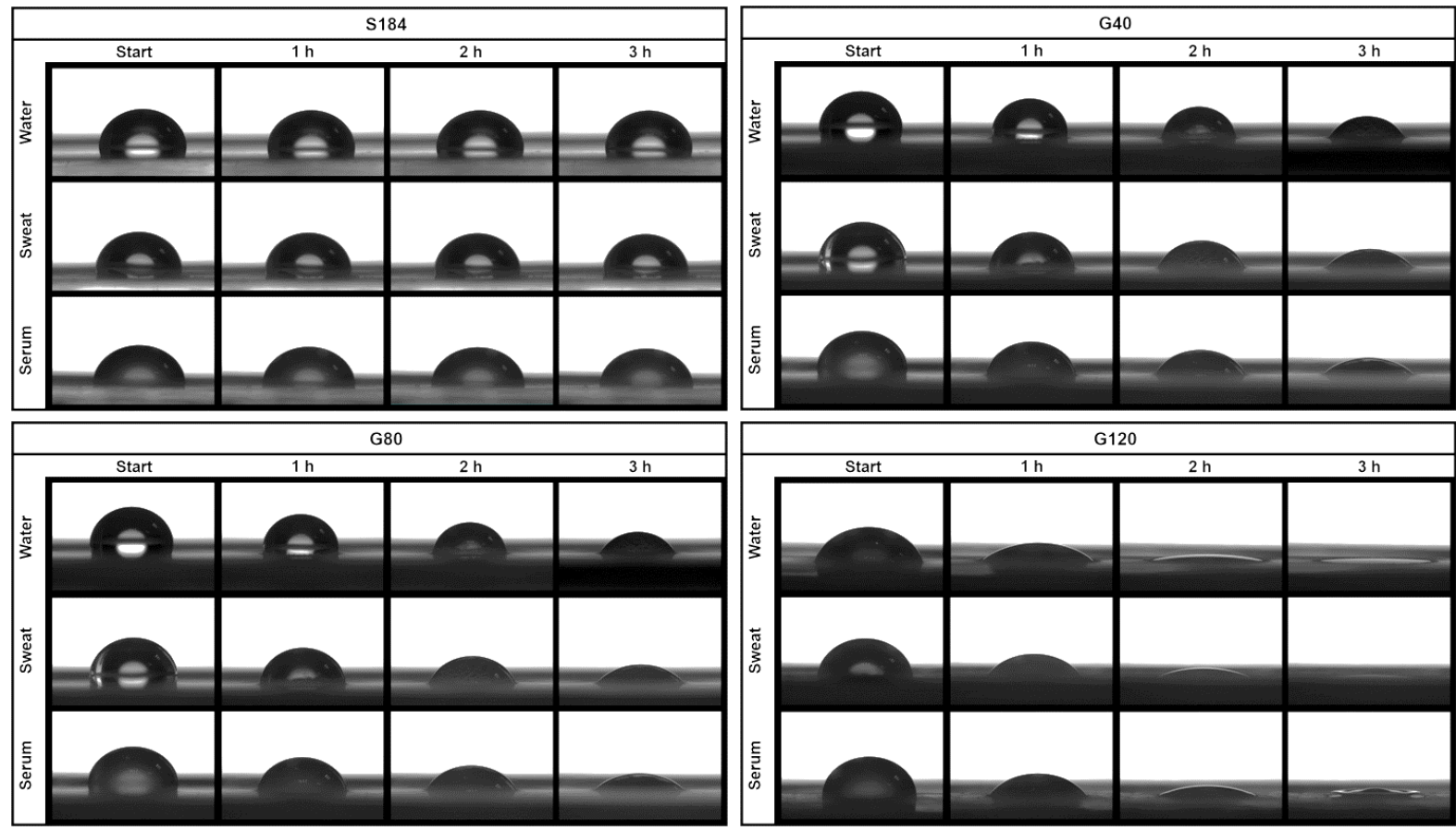

Figure 4. Progressive absorption of DI water, artificial sweat and human blood serum by glycerol-silicone membranes containing $0,40,80$ and $120 \mathrm{phr}$ of glycerol. Images were taken at the start of the experiment and again after 1,2 and 3 hours.

\subsection{Release of various drugs from the glycerol-silicone membranes}




\section{WILEY-VCH}

Glycerol-silicone elastomers have been reported to enable delivery of substances upon exposure to an aqueous environment ${ }^{[37]}$, and this achieved mass transport has been attributed to the concentration gradient between the glycerol phase of the elastomer and the aqueous environment. ${ }^{[45]}$ In GSEs, the silicone phase surrounding each glycerol domain acts as a semipermable membrane, allowing small molecules to migrate out of the material. ${ }^{[45]}$ Moreover, it has been shown that the release kinetics of this migration can be precisely controlled by adjusting the glycerol loading of the elastomer. Elastomers with 40, 80 and $120 \mathrm{phr}$ of glycerol exhibit first-, near zero- and zero-order release kinetics, respectively. ${ }^{[37]}$ In this study, release profiles of more complex molecules, such as various antimicrobial and anti-inflammatory drugs, were investigated. Substances were selected based on their potential usefulness in wound care products, and samples containing $80 \mathrm{phr}$ of glycerol were studied unless otherwise specified; this glycerol concentration was previously reported to result in the release of hydroquinone (investigated active substance) following near zero-order kinetics, with delivery rates intermediate between compositions with 40 and $120 \mathrm{phr}$ of glycerol (120 phr of glycerol defines the higher limit of glycerol loading, at which the membranes possess excellent mechanical integrity). The mechanism of drug delivery for a representative antimicrobial agent is presented in Figure 5. 


\section{WILEY-VCH}
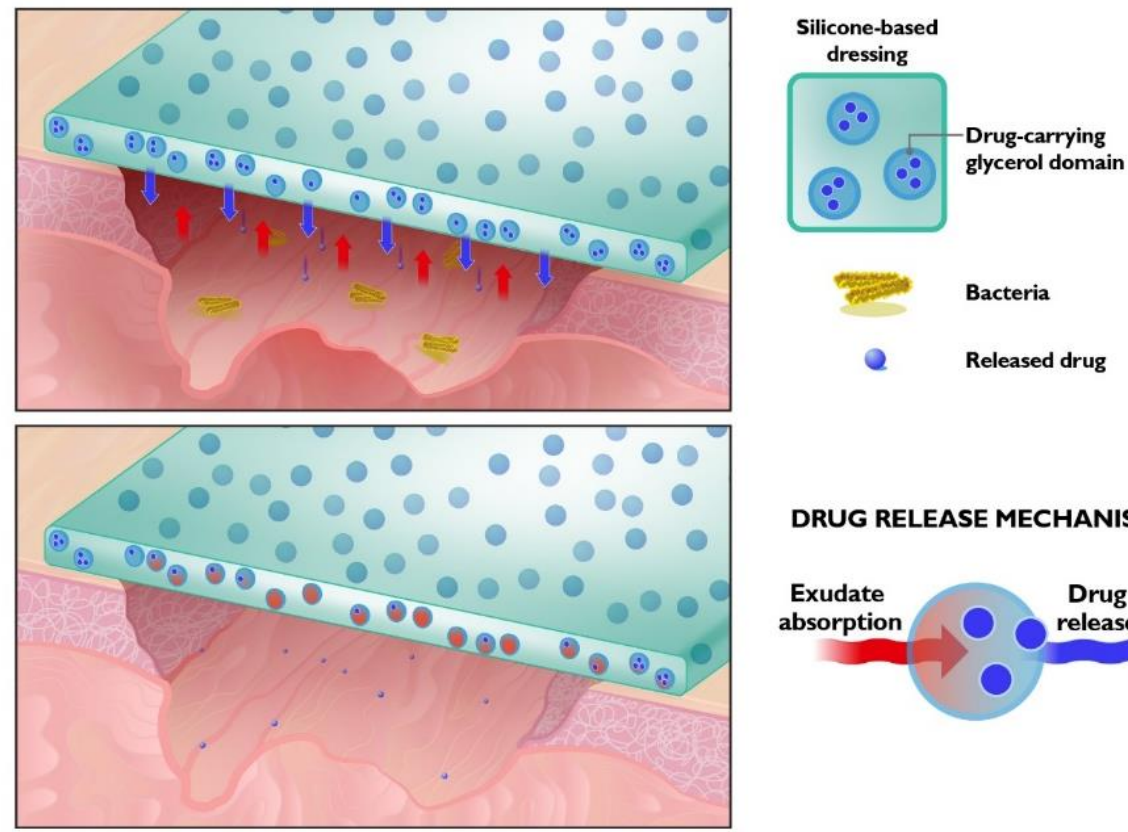

DRUG RELEASE MECHANISM

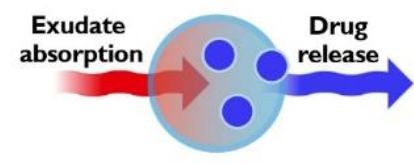

Figure 5. Schematic depiction of the antimicrobial drug delivery action from a glycerol-silicone membrane to an infected wound. The membrane is placed over an infected wound; the wound exudate is then absorbed by the membrane while the antimicrobial drug is simultaneously released to the wound bed to eliminate the existing biofilm and prevent future infections.

\subsubsection{Morphology of glycerol-silicone elastomer with and without incorporated drugs}

Figure 6 shows SEM images of cross-sections of $80 \mathrm{phr}$ glycerol compositions containing no drug, tetracycline and triclosan- $\beta C D$ complex, respectively. Drug-free samples and those with incorporated tetracycline exhibit nearly identical morphology with comparable droplet size, while the sample containing triclosan- $\beta C D$ complex has slightly smaller droplets. Cyclodextrins contain both hydrophilic and hydrophobic functional groups and thus act as surfactants, decreasing the interfacial tension between the silicone and glycerol phases and eventually leading to a decrease in droplet diameter. Similar behavior was previously observed when a polysorbate-based surfactant was incorporated into the GSEs. ${ }^{[38]}$ 


\section{WILEY-VCH}
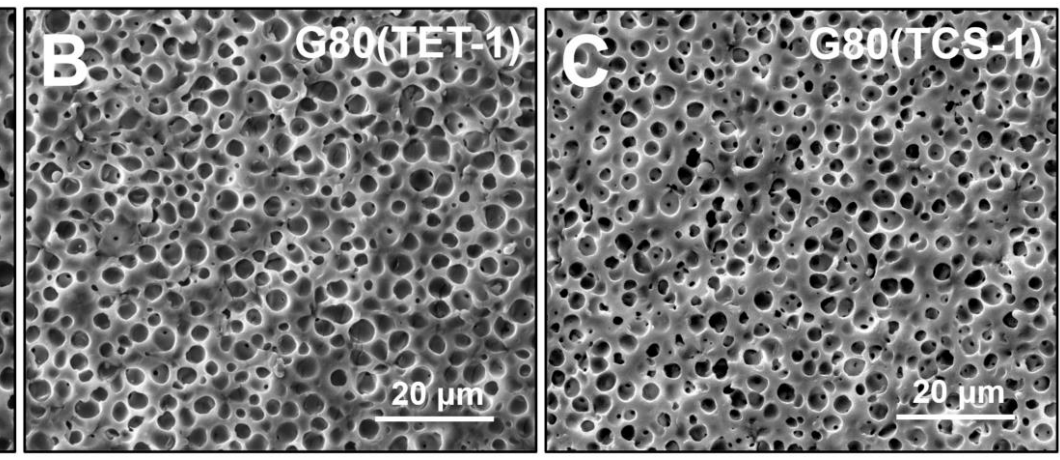

Figure 6. Scanning electron microscopy images of cross-sections of cross-linked G80

samples with no drug (A), with tetracycline (B) and with triclosan- $\beta C D$ complex (C).

\subsubsection{Optimization of the release experiment and release behavior of tetracycline}

Tetracycline (TET) was first thoroughly investigated, since this antimicrobial drug has been reported to be a promising candidate for treating infected wounds. ${ }^{[1,46,47]}$ Drug release experiments were performed using the setup presented in Figure 7A. Each glycerol-silicone membrane containing a drug was inserted into a quartz cuvette, and the drug release was monitored until no further increase in absorbance was observed. Detailed information on drug concentration and mass per sample area can be found in Table S1 in the Supporting Information. To begin, the influence of the release process on both the type of recipient medium and the drug concentration in the glycerol phase was investigated. The results indicate that the release profiles of TET to DI water and to saline water, respectively, are nearly identical (see Figure 7B). Moreover, as shown in Figure 7C, the drug content of the membrane does not substantially influence the release rate, as the percentage TET release rates from compositions with $0.5,1$ and $2 \mathrm{wt} \%_{-}$-of the drug in the glycerol phase (corresponding to 74, 148 and $296 \mu \mathrm{g}$ of the drug per $\mathrm{cm}^{2}$ of the sample, respectively) were comparable. Importantly, this indicates that, by adjusting the drug concentration (and thus the total drug load) in the glycerol phase of the membrane, the drug delivery dose can be easily tuned without influencing delivery kinetics. Based on the results presented in Figure 7B-C, it was decided to use saline water as the drug 


\section{WILEY-VCH}

recipient medium and to keep the drug concentration in glycerol at $1 \mathrm{wt} \%$ for all further experiments.

The hypothesis that glycerol loading can be used as a tool to tune both the rate and kinetics of drug delivery is confirmed by the results presented in Figure 7D, which show that TET was released most slowly by sample G40 and fastest by sample G100. Interestingly, sample G40 released very low amounts of the drug (relative to the total content in the membrane) compared to samples G80 and G100. The previously observed differences in the release of hydroquinone (a substantially smaller molecule than TET) were not as substantial ${ }^{[37]}$, indicating that the size of the released substance influences the final release profile, with larger molecules being released more slowly from the GSE membranes. Importantly, these experiments proved that GSE membranes can efficiently deliver substances as large as TET (structural formula of TET in Figure 7B).

A

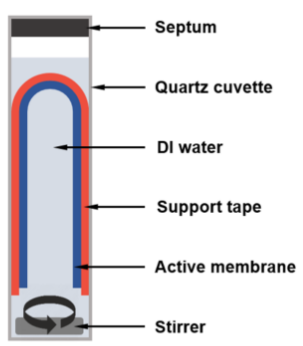

B
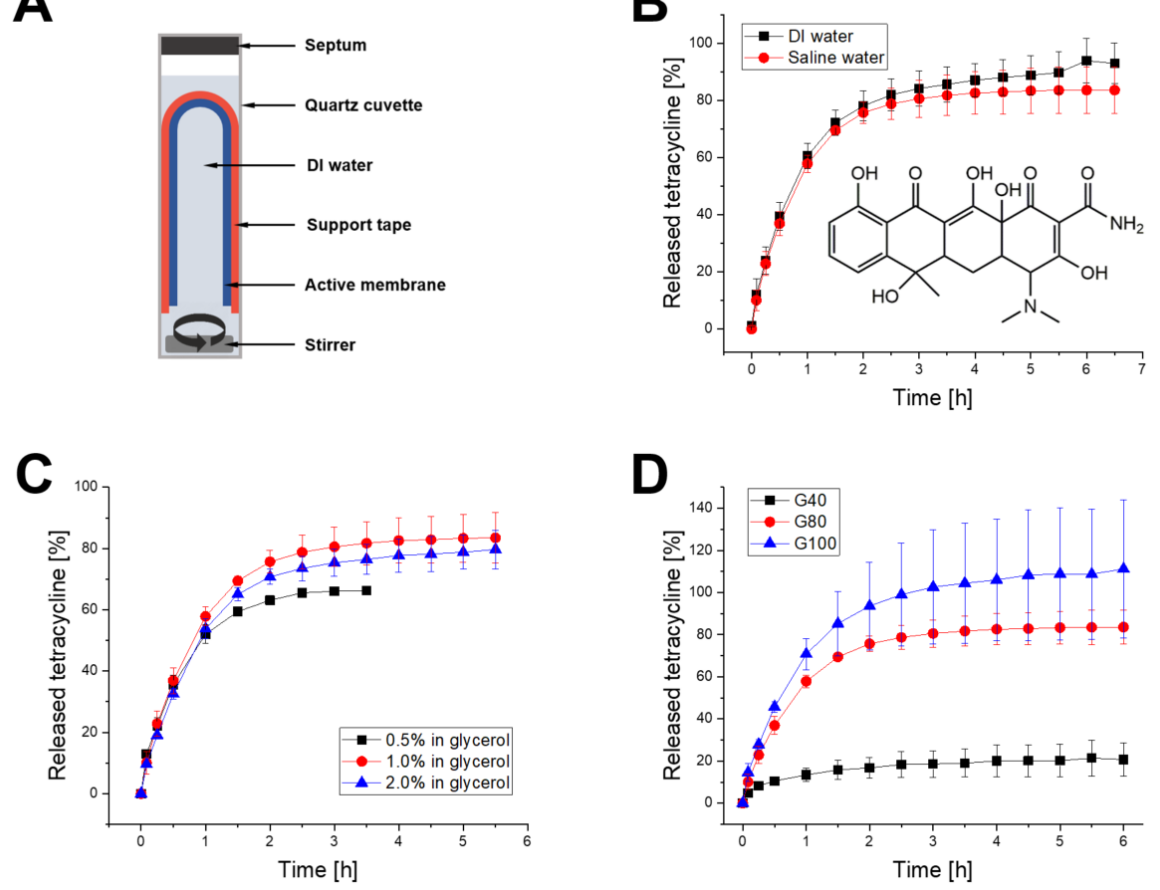

Figure 7. (A) Schematics of drug release experiment setup. A GSE membrane is inserted into a quartz cuvette equipped with a stirrer. The cuvette is then filled with saline solution and 


\section{WILEY-VCH}

inserted into a UV-vis spectrophotometer to enable online tracking of substance release. (B) Release profiles of tetracycline to DI water and saline water, respectively. (C) Release profiles of tetracycline from G80 compositions containing $0.5,1$ and $2 \mathrm{wt} \%$ of drug dissolved in the glycerol phase. (D) Release profiles of tetracycline from compositions G40, G80 and G100.

\subsubsection{Release behavior of other substances}

Release profiles of several other substances from G80 compositions were investigated to verify GSE membrane versatility with respect to drug delivery. Results of these release experiments are presented in Figure 8.

Our findings showed that all investigated substances were released from the membranes, albeit at different release rates. Interestingly, TCS, IBU, DCF and LDC were released by near zeroorder kinetics, as expected based on results obtained previously. ${ }^{[37]}$ The substances were delivered at an approximately constant rate in the first part of the process (up to around 2/3 of the total release), after which substance release decelerated. The first-order kinetics observed for the specimens containing MUP and BKC confirmed that molecule size has a substantial impact on release behavior. Generally, smaller molecules are delivered faster and at a more constant rate, although more factors are believed to influence the final release profiles: e.g., drug polarity. It is important to stress that no burst release (rapid discharge of substances at the beginning of the release process) of the kind that is often encountered in drug delivery devices was observed in any of the cases studied here. ${ }^{[8]}$ The results further indicate that some drugs are not completely released from the membranes-i.e., that the release profiles reach a plateau below $100 \%$ of the theoretical maximum release. Because the majority of the investigated substances have very limited solubility in glycerol and even weaker solubility in water, it is indicating that this incomplete release is caused by poor drug solubility in the recipient medium. This is arguably of minimal concern in the case of drug delivery to wounds, however, since the released drug will be bound by the bacteria or cells, enabling further release of the active 


\section{WILEY-VCH}

substance to the wound exudate and thereby eventually changing the partition coefficients in favor of releasing more drug.

The results discussed above confirm that GSE membranes are a universal platform for the delivery of active substances, including those that are not soluble in hydrophilic media. Importantly, the fact that the drugs are already dissolved in the glycerol domains of the membranes is considered to be one of the main advantages of this system. Substances in the dissolved state can be readily released from a membrane, while the silicone phase encapsulating each glycerol domain ensures sustained and controlled drug dosage. GSE membrane technology thus allows for precise control of the release profile, delivery dose and delivery period - all of which can be tuned by adjusting the glycerol loading in the membranes, drug concentration in the glycerol phase or membrane thickness. ${ }^{[37]}$

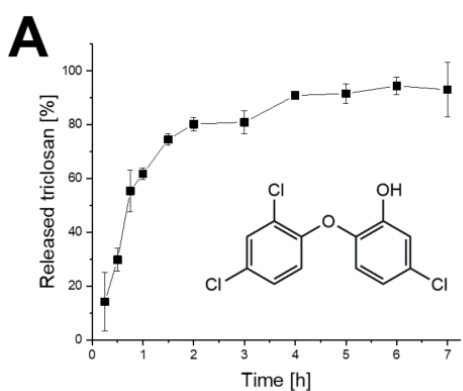

B



E

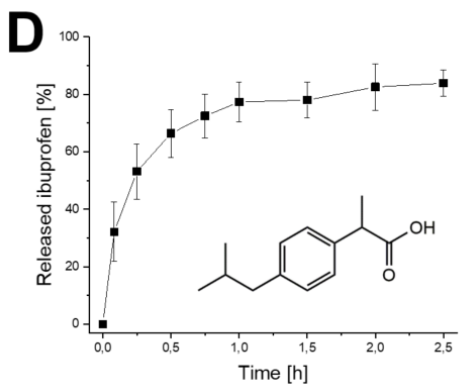

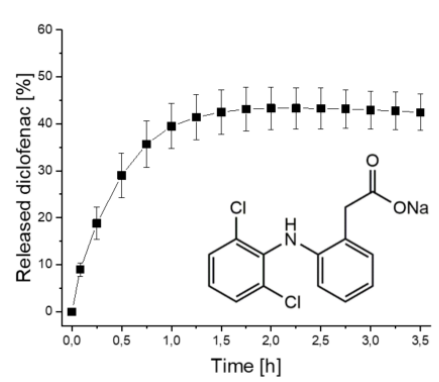

C

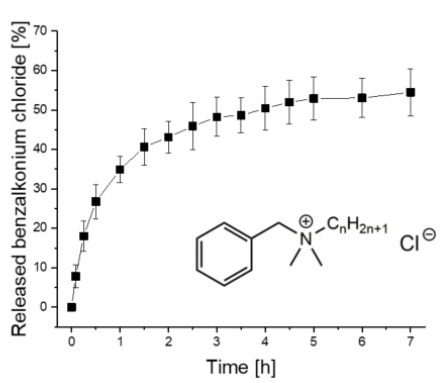

$\mathbf{F}$

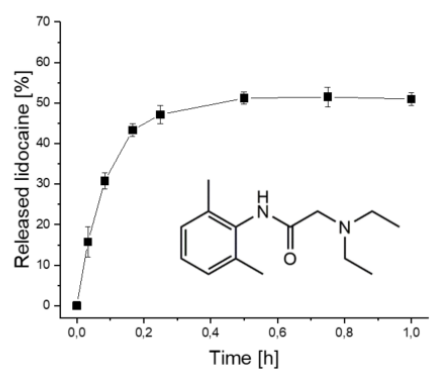

Figure 8. Release profiles of triclosan (A), mupirocin (B), benzalkonium chloride (C), ibuprofen (D), diclofenac (E) and lidocaine (F).

\subsection{Antimicrobial activity of the GSE membranes}




\section{WILEY-VCH}

To assess the antimicrobial activity of the GSE membranes, we determined the antimicrobial susceptibility of ten bacterial strains towards G80(TCS-1) using agar diffusion assays (Figure 9A). These assays replicate a wound environment by placing the bacteria on a surface which contains nutrients for bacterial proliferation. We found that G80(TCS-1) inhibited all bacterial strains except $P$. aeruginosa PAO1, which is known to be resistant to triclosan and hence served as a negative control (Figure 9B). ${ }^{[48]}$ The high reproducibility of these results confirms the reliability of drug release from glycerol-silicone membranes even at low moisture conditions such as those present in the agar diffusion assay, which arguably simulate low exudate conditions in wounds.

For comparison, we also examined the antimicrobial effect of two commercial wound dressings, Biatain Alginate Ag (dressing for medium-to-high exudate wounds) and Mepilex Ag (dressing for low-to-medium exudate wounds), in the same experimental setup. It was found out that Biatain consistently inhibited the growth of $S$. pyogenes and occasionally inhibited the growth of five other bacteria (Figure 9C). In contrast, none of the ten bacterial strains exhibited susceptibility to Mepilex. However, it is possible that the moisture level present in the agar diffusion assay was not sufficient to reveal the antimicrobial potential of the commercial dressings.

To assess the performance of the dressings at conditions with high water levels-simulating (such as those present in highly exuding woundshigh exudate levels), we saturated the dressings by pre-soaking them in $0.5 \mathrm{~mL}$ of $0.9 \% \mathrm{NaCl}$ solution before placing them on the agar surface. The GSEs were treated and tested in the same way for comparison. As expected, the performance of pre-soaked Biatain samples improved compared to dry samples: the pre-soaked Biatain was able to inhibit the growth of eight out of ten bacterial strains. However, the presoaked Mepilex did not inhibit any of the bacteria tested (for more details, see Table $\mathrm{S} \pm \underline{2}$ in the Supporting Information). 


\section{WILEY-VCH}

Next, in order to simulate a system with even higher exudate-water levels, a liquid-based assay was used. In this test, no growth of the investigated bacteria (A. baumannii) was observed in the presence of the Mepilex samples, indicating successful inhibition similar to that reported in previous studies using liquid-based assays. ${ }^{[49]}$ This suggests that excessive amounts of exudate water-based media are required to extract or activate the antimicrobial silver from the Mepilex dressing in order to trigger the inhibition of bacterial growth. The investigated Mepilex dressing is composed of a drug-containing absorbent foam coated with a porous adhesive. It is therefore possible that products with similar designs require more prominent stimuli-e.g., high water or exudate content combined with periodic deformation or pressure - to fully achieve their drug delivery potential.

The GSE with incorporated triclosan exhibited consistently high antimicrobial efficacy at both low and high levels of exudatewater, and its bacteria inhibition zones were substantially larger in the case of all investigated strains (with the exception of $P$. aeruginosa, for the reasons mentioned above) compared to Biatain and Mepilex. Thus, the results clearly demonstrate that GSE membranes outperformed the commercial dressings studied here in terms of drug release and, hence, antimicrobial efficacy even at low exudate-moisture levels. 


\section{WILEY-VCH}

A


Figure 9. Antimicrobial susceptibility testing. (A) Left: Schematic of the experimental setup for determining the antimicrobial susceptibility of GSE, Biatain and Mepilex dressings. The petri dish has a diameter of $14 \mathrm{~cm}$, and the individual dressing samples have a diameter of 6 mm. Right: A representative experimental result: a photograph for S. aureus NCTC 8325-4 after incubation and exposure to G0 (no glycerol), G80 (80 phr glycerol), G80(TCS-1) (1\% Triclosan), Biatain Ag, and Mepilex Ag samples. (B) Inhibition zone sizes (in mm) obtained for G80(TCS-1) and ten bacterial strains. The data were obtained from three independent experiments with triplicates (= nine data points). (C) Inhibition zone sizes (in mm) obtained for Biatain $\mathrm{Ag}$ and ten bacterial strains. The data were obtained from three independent experiments in triplicates (= nine data points).

\section{Conclusions}




\section{WILEY-VCH}

The addition of glycerol microdroplets to silicone elastomers was proven to enhance the material's water vapor transmission rate by up to $200 \%$ in addition to enabling the absorption of liquids such as DI water, sweat and blood serum. The absorption rate was comparable for all investigated liquids, and generally increased with increased glycerol loading. Interestingly, absorption takes place despite the GSEs' hydrophobic surface. While GSEs' Young's moduli decreased with increasing glycerol content, the ultimate strain was minimally affected up to glycerol loadings of $80 \mathrm{phr}$, proving that the material's mechanical performance remains uncompromised despite substantial volumetric dilution by glycerol. What is more, the versatility of GSE technology was demonstrated by introducing a wide variety of drugs (e.g., antimicrobial and anti-inflammatory agents) into the glycerol domains, from which these drugs can then be released in a controlled fashion. Release kinetics can be varied between first- and zero-order by controlling the glycerol loading, while the rate of release can be tuned by adjusting the drug concentration in glycerol. Drug release is triggered by minimum amounts of moisture (as shown via an agar diffusion assay study), and the delivered drugs efficiently inhibit bacterial growth. The efficient discharge of drugs from GSEs could be vital in situations requiring immediate cutaneous drug therapy. Glycerol-silicone materials constitute a robust and versatile technology with multiple potential wound care applications, such as individual elastomeric dressings — e.g., for burns — or as the adhesive component of more complex wound care dressings containing fiber or foam absorbers.

\section{Supporting Information}

Supporting Information is available from the Wiley Online Library or from the author.

\section{Acknowledgements}




\section{WILEY-VCH}

The authors are thankful for the generous funding from Independent Research Fund Denmark and Innovation Fund Denmark for the Innoexplorer grant. Special gratitude goes to Seonghyeon Jeong for help with graphic design.

\section{Conflict of Interest}

The authors declare no conflict of interest.

Received: ((will be filled in by the editorial staff))

Revised: ((will be filled in by the editorial staff)) Published online: ((will be filled in by the editorial staff))

\section{References}

[1] A. S. Boateng, K. H. Matthews, H. N. E. Stevens, G. M. Eccleston, J. Pharm. Sci. 2008, 97, 2892.

[2] M. C. Ferreira, P. Tuma, V. F. Carvalho, F. Kamamoto, Clinics 2006, 61, 571.

[3] K. Järbrink, G. Ni, H. Sönnergren, A. Schmidtchen, C. Pang, R. Bajpai, J. Car, Syst. Rev. 2017, 6, 1 .

[4] J. Posnett, P. J. Franks, Nurs. Times 2008, 104, 44.

[5] D. R. Bickers, H. W. Lim, D. Margolis, M. A. Weinstock, C. Goodman, E. Faulkner, C. Gould, E. Gemmen, T. Dall, J. Am. Acad. Dermatol. 2006, 55, 490.

[6] J. G. Powers, C. Higham, K. Broussard, T. J. Phillips, J. Am. Acad. Dermatol. 2016, $74,607$.

[7] C. K. Sen, G. M. Gordillo, S. Roy, R. Kirsner, L. Lambert, T. K. Hunt, F. Gottrup, G.

C. Gurtner, M. T. Longaker, Wound Repair Regen. 2009, 17, 763.

[8] J. Boateng, O. Catanzano, J. Pharm. Sci. 2015, 104, 3653.

[9] J. G. Powers, L. M. Morton, T. J. Phillips, Dermatol. Ther. 2013, 26, 197. 


\section{WILEY-VCH}

[10] Y. Zhu, J. Zhang, J. Song, J. Yang, T. Xu, C. Pan, L. Zhang, J. Mater. Chem. B 2017, 5,8451 .

[11] S. Dhivya, V. V. Padma, E. Santhini, BioMedicine 2015, 5, 24.

[12] M. Zilberman, D. Egozi, M. Shemesh, A. Keren, E. Mazor, M. Baranes-Zeevi, N. Goldstein, I. Berdicevsky, A. Gilhar, Y. Ullmann, Acta Biomater. 2015, 22, 155.

[13] C. D. Roberts, D. J. Leaper, O. Assadian, Adv. Wound Care 2017, 6, 63.

[14] M. Shemesh, M. Zilberman, Acta Biomater. 2014, 10, 1380.

[15] S. L. Percival, D. Mayer, A. M. Salisbury, Wound Repair Regen. 2017, 25, 767.

[16] B. A. Lipsky, E. J. G. Peters, E. Senneville, A. R. Berendt, J. M. Embil, L. A. Lavery, V. Urbančič-Rovan, W. J. Jeffcoate, Diabetes Metab. Res. Rev. 2012, 28, 163.

[17] A. Kramer, J. Dissemond, S. Kim, C. Willy, D. Mayer, R. Papke, F. Tuchmann, O. Assadian, Skin Pharmacol. Physiol. 2018, 31, 28.

[18] J. S. Glasser, Burns 2014, 21, 7251.

[19] S. O’Meara, D. Al-kurdi, Y. Ologun, L. G. Ovington, J. M. Martyn-St, R. R, Cochrane Database Syst. Rev. 2014, 1,1 .

[20] X. Zhao, H. Wu, B. Guo, R. Dong, Y. Qiu, P. X. Ma, Biomaterials 2017, 122, 34.

[21] R. J. White, J. Wound Care 2009, 18, 245.

[22] I. Cantón, R. Mckean, M. Charnley, K. A. Blackwood, C. Fiorica, A. J. Ryan, S. MacNeil, Biotechnol. Bioeng. 2010, 105, 396.

[23] V. Arapoglou, K. Katsenis, K. N. Syrigos, E. P. Dimakakos, N. Zakopoulou, D. Tsoutsos, K. Gjødsbøl, C. Glynn, E. Schäfer, B. Petersen, J. Wound Care 2011, 20, 319.

[24] B. Jørgensen, G. J. Friis, F. Gottrup, Wound Repair Regen. 2006, 14, 233.

[25] M. Romanelli, V. Dini, R. Polignano, P. Bonadeo, G. Maggio, J. Dermatolog. Treat. 2009, 20, 19.

[26] J. L. Lázaro-Martínez, F. J. Álvaro-Afonso, D. Sevillano-Fernández, R. J. Molines- 


\section{WILEY-VCH}

Barroso, Y. García-Álvarez, E. García-Morales, Int. J. Low. Extrem. Wounds 2019, 18, 269.

[27] P. Mazurek, S. Vudayagiri, A. L. Skov, Chem. Soc. Rev. 2019, 48, 1448.

[28] K. Mojsiewicz-Pieńkowska, M. Jamrógiewicz, M. Zebrowska, B. Mikolaszek, M. Sznitowska, Int. J. Pharm. 2015, 481, 18.

[29] B. Bleasdale, S. Finnegan, K. Murray, S. Kelly, S. L. Percival, Adv. Wound Care 2015, $4,422$.

[30] R. Xu, G. Luo, H. Xia, W. He, J. Zhao, B. Liu, J. Tan, J. Zhou, D. Liu, Y. Wang, Z. Yao, R. Zhan, S. Yang, J. Wu, Biomaterials 2015, 40, 1.

[31] R. Xu, H. Xia, W. He, Z. Li, J. Zhao, B. Liu, Y. Wang, Q. Lei, Y. Kong, Y. Bai, Z. Yao, R. Yan, H. Li, R. Zhan, S. Yang, G. Luo, J. Wu, Sci. Rep. 2016, 6, 1.

[32] Z. Li, A. Milionis, Y. Zheng, M. Yee, L. Codispoti, F. Tan, D. Poulikakos, C. H. Yap, Nat. Commun. 2019, 10, 1.

[33] W. Du, Z. Zhang, Z. Li, Polym. Int. 2019, 68, 1739.

[34] S. Wei, Y. You, Y. Ma, W. Huang, X. Liang, A. Zhang, Y. Lin, React. Funct. Polym. 2019, 141, 21.

[35] J. Zhao, G. Luo, J. Wu, H. Xia, ACS Appl. Mater. Interfaces 2013, 5, 2040.

[36] P. Mazurek, S. Hvilsted, A. L. Skov, Polymer 2016, 87, 1.

[37] P. Mazurek, M. A. Brook, A. L. Skov, Langmuir 2018, 34, 11559.

[38] P. Mazurek, L. Yu, R. Gerhard, W. Wirges, A. L. Skov, J. Appl. Polym. Sci. 2016, 133, 1.

[39] P. Mazurek, B. E. F. Ekbrant, F. B. Madsen, L. Yu, A. L. Skov, Eur. Polym. J. 2019, $113,107$.

[40] V. Chiaula, P. Mazurek, J. Eiler, A. C. Nielsen, A. L. Skov, Int. J. Adhes. Adhes. 2020, $102,102667$.

[41] J. Trifol, D. Plackett, C. Sillard, O. Hassager, A. E. Daugaard, J. Bras, P. Szabo, J. 


\section{WILEY-VCH}

Appl. Polym. Sci. 2016, 133, 1.

[42] J. Trifol, D. Plackett, C. Sillard, P. Szabo, J. Bras, A. E. Daugaard, Polym. Int. 2016, $65,988$.

[43] R. W. Style, R. Boltyanskiy, B. Allen, K. E. Jensen, H. P. Foote, J. S. Wettlaufer, E. R. Dufresne, Nat. Phys. 2015, 11, 82.

[44] J. d. Eshelby, Proc. Phys. Soc. London, Ser. A 1957, 241, 376.

[45] P. Mazurek, P. M. Zelisko, A. L. Skov, M. A. Brook, ACS Appl. Polym. Mater. 2020, 2, 1203.

[46] T. R. S. Kumar, M. V. Bai, L. K. Krishnan, Biologicals 2004, 32, 49.

[47] H. Chen, X. Xing, H. Tan, Y. Jia, T. Zhou, Y. Chen, Z. Ling, X. Hu, Mater. Sci. Eng. C 2017, 70, 287.

[48] L. Zhu, J. Lin, J. Ma, J. E. Cronan, H. Wang, Antimicrob. Agents Chemother. 2010, 54, 689.

[49] P. Davies, S. McCarty, K. Hamberg, J. Wound Care 2017, 26, S1. 


\section{WILEY-VCH}

Table of Content

Glycerol-silicone membranes represent a platform technology for controlled delivery of actives substances to wounds. They enable release of virtually any drug such as antimicrobial, antiinflammatory or pain relief agents and thus enable treatment of various disorders. Here we show that the membranes are an efficient tool in fighting bacterial infections outperforming commercial state-of-the-art wound dressings.

Piotr Mazurek, Nikoline S. Frederiksen, Harald Silau, Nuura A. Yuusuf, Hanne Mordhorst, Sünje J. Pamp, Anne L. Skov*

\section{Glycerol-silicone membranes for sustained and controlled topical delivery of antimicrobial and pain-relief drugs}

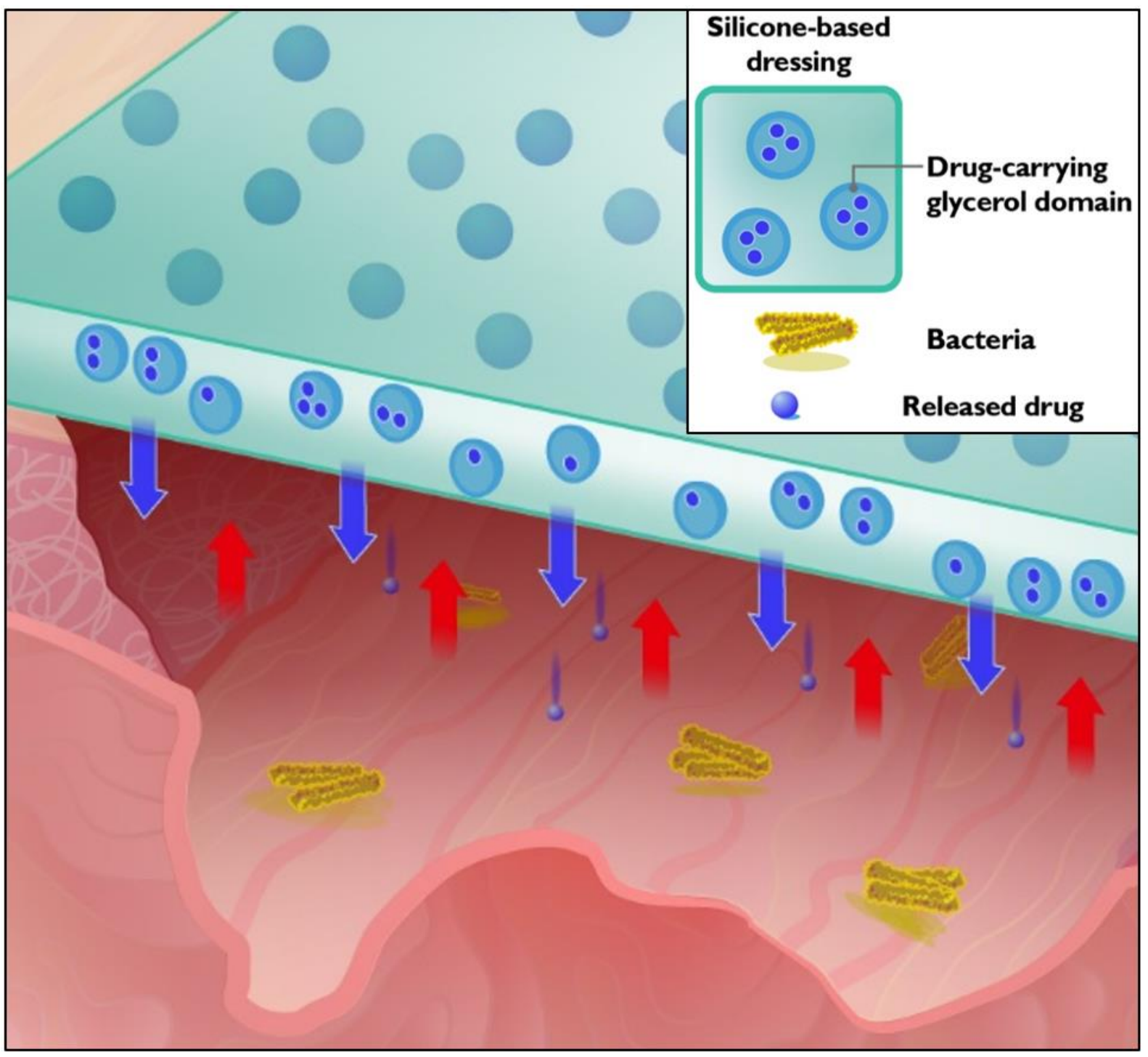


Click here to access/download

Production Data

Release of drugs from GSEs.7z 